An Official Publication of IGM Publication

\title{
Moment Scheme of the Finite Element for Spatially Reinforced Composite Materials
}

\author{
Authors \\ Sergij Grebenyuk*, Olha Titova** \\ *Associate Professor, Ph.D. in Engineering, Zaporizhzhya National University, Zhukovsky 66, 69600 Zaporizhzhya, Ukraine, \\ Email: gsm1212@ukr.net \\ **Associate Professor, Ph.D. in Engineering, Zaporizhzhya National University, Zhukovsky 66, 69600 Zaporizhzhya, Ukraine,
} Email:toa7676@gmail.com

\section{ABSTRACT}

The paper presents the computational problems of application of the spatial finite element method for the calculation of constructions made of fiber-reinforced composites. Construction of stiffness matrix of a 3-dimentional finite element is performed taking into account the fibers' spatial orientation. The moment scheme of the finite element is applied for improving results convergence. It is based on double approximation of displacement fields and deformation components and retaining some number of addends according to the definite rule. This approach is applied to the deformation problem of a hollow cylinder made of composite material. Numerical calculation results have shown good matching with analytical solutions.

Keywords: moment scheme, spatially fiber-reinforced composite materials, stiffness matrix.

\section{INTRODUCTION}

Determining mechanical characteristics of spatially reinforced composites is one of the most important tasks of modern mechanics of composite materials. Structural analysis of fiber composites has mathematical problems. These fiber composites exhibit anisotropy of the elastic properties. In addition, the characteristics of the fiber-reinforced composite may have a complex geometry. Therefore, the analytical calculation is possible only for simple composite structures. The numerical methods are applied to analysis of complex structures. In this paper the finite element method with modifications is considered.

In the calculations, as a rule, composite is represented as a homogeneous isotropic (transtropic) material. After this the problem is solved for the orthotropic material.

Application of finite element method to solve such problems has been investigated in many papers, some of them ${ }^{[1-10]}$. The various mechanical theories and the various dimension of the problem can be used to construct the finite elements. The onedimensional, two-dimensional, spatial problems are considered in researches ${ }^{[2,3,5,9]}$.

For more accurate results based on the finite element method different approaches are used. Thus, polynomials of higher order or special scheme are used in the approximation. To calculate the composite structures have been proposed various types of finite elements. The paper [9] is devoted to application of semi-analytical method of finite elements. The moment schema of the finite element is considered in the papers ${ }^{[1,9,10]}$.

The purpose of this paper is the determination of the stiffness matrix of a composite with randomly-oriented fibers by the moment scheme of the finite element.

\section{BASIC RELATIONS}

Basic relations are represented for the stiffness matrix of the spatial finite element of the transversely isotropic material based on the moment scheme.

Consider three coordinate systems (basic system and two local systems). Each of these systems has advantages. Thus, the basic coordinate system is unchanged. All the geometrical and mechanical characteristics of the structure, boundary and initial conditions are defined in this system. The basic system is Cartesian coordinate system. The first local coordinate system is a nonorthogonal curvilinear coordinate system associated with the finite element. The origin is the center of the finite element. The direction of the axes is associated with direction edges of the finite element. The coefficients of the stiffness matrix of the finite element are calculated in this system. The second local coordinate system associated with the direction of the reinforcement fibers. The elastic constants of the material, the components of the tensor of strains and stresses are calculated in this coordinate system. If you use the traditional approach to the finite element method to determine the stress-strain state spatial structures seen a number of negative effects: neglect displacement finite element as absolutely rigid body and the effect of "false shift." The first phenomenon is manifested in the slow convergence of the numerical results, if the received version of the approximation of displacements not accurately describe the displacement of the finite element as a rigid body [3-4]. The second phenomenon is that when bending thin plates and shells by spatial finite element significantly increases the errors associated with the appearance of a fictitious shear strains ${ }^{[2]}$.

To eliminate these negative phenomena has been developed so-called moment scheme of finite element ${ }^{[2]}$. The basic idea of this scheme is as follows: the components of the displacement field and held a number of initial terms, similarly expanded in a number of the components of the strain tensor and slightly compressible material changes in the volume function are decomposed in series. After that, the correspondence of the last two expansions to displacement expansion is checked. If the expansion includes terms that are not in the displacement expansion, the corresponding terms are removed from the decomposition. Thus decomposition is used for obtaining the stiffness matrix of the finite element. 
One of the most widely used finite elements obtained during a sampling spatial construction is parallelepiped finite element.

Consider determining a stiffness matrix in the local coordinate system associated with the finite element (Fig. 1). In this system, the curvilinear parallelepiped element is mapped in the isoparametric finite element in the form of a cube with an edge length of two. We place the origin of the local coordinate system in the center of the cube. Axis are directed along the edges of the cube.

Consider the local coordinate system associated with the direction of reinforcing fiber material (Fig. 1). We place the origin of this system in the center of the cube. The fiber is randomly oriented in space. The direction of the reinforcement fibers in the coordinate system of a finite element defined by two angles: $\alpha$ and $\beta$. Unlike most of the proposed finite elements the reinforcement is determined in planes parallel to the faces of the finite element. This narrows the class of tasks or leads to large inaccuracies in the calculation. When discretising the stringent requirements arise for alignment of reinforcement direction with grid lines direction. This limits the possibility of automatic mesh generation.

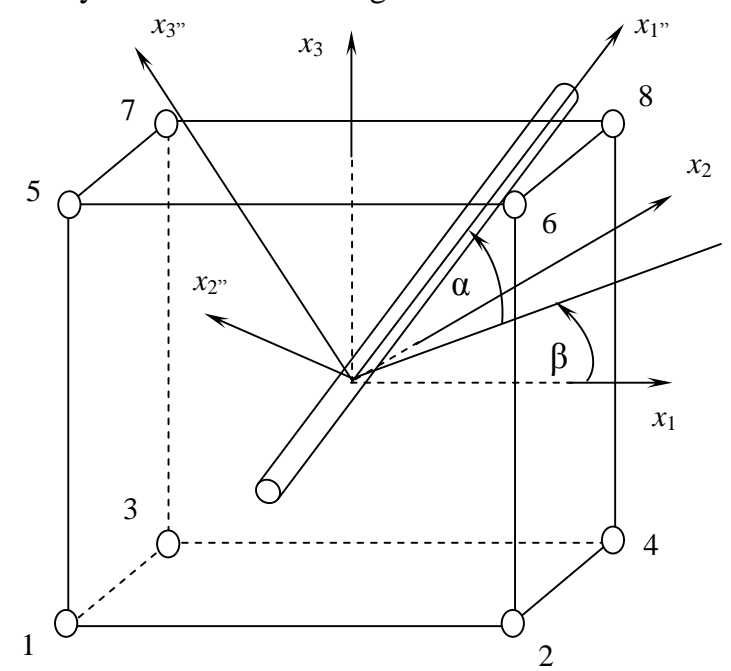

Fig. 1 Finite element with taking into account the fibers's spatial orientation

We use a variation approach in the derivation of the fundamental relations of the stiffness matrix. Consider the variation of the elastic deformation energy of the finite elements:

$$
\delta W=\iiint_{V} \sigma^{i j} \delta \varepsilon_{i j} d V
$$

or

$$
\delta W=\iiint_{V} C^{i j k l} \varepsilon_{k l} \delta \varepsilon_{i j} d V
$$

In matrix form it can be written, as

$$
\delta W=\iiint_{V}\left\{\delta \varepsilon_{i j}\right\}^{T}\left[C^{i j k l}\right]\left\{\varepsilon_{k l}\right\} d V
$$

where $\left\{\varepsilon_{k l}\right\}=\left\{\varepsilon_{11}, \varepsilon_{12}, \varepsilon_{13}, \varepsilon_{21}, \varepsilon_{22}, \varepsilon_{23}, \varepsilon_{31}, \varepsilon_{32}, \varepsilon_{33}\right\}^{T}$ is the vector of strain, [C $\left.C^{i j k l}\right]$ is the matrix of elastic constants of an isotropic body in the coordinate system of the finite element $x_{i}$. The components of the tensor of the elastic constants of the most convenient to calculate in the coordinate system of reinforcement $x_{m^{\prime \prime}}$. Fiber composites have transtropic properties. Isotropy plane is perpendicular to the fiber axis. Using the symmetry of the tensor of the elastic constants and the strain tensor can be written as $\left\{\varepsilon_{k l}\right\}=\left\{\varepsilon_{11}, \varepsilon_{22}, \varepsilon_{33}, \varepsilon_{12}, \varepsilon_{13}, \varepsilon_{23}\right\}^{T}$,

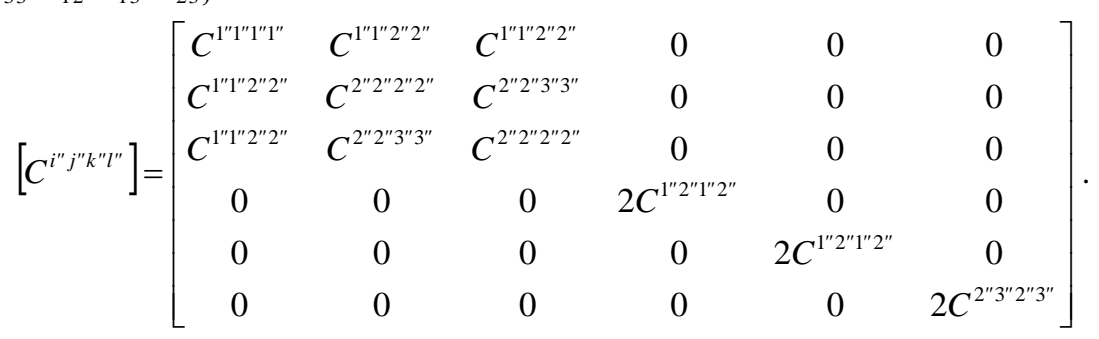

Components of the tensor of the elastic constants $C^{i " j j^{\prime \prime}} l^{\prime \prime}$ for orthotropic body in the coordinate reinforcement system are calculated using the following equations ${ }^{[5]}$ :

$$
\begin{aligned}
C^{1^{\prime \prime} 1^{\prime \prime} 1^{\prime \prime} 1^{\prime \prime}}=\frac{E_{1}}{\Delta_{*}}\left(1-v_{23} v_{32}\right) ; C^{1^{\prime \prime} 1^{\prime \prime 2} 2^{\prime \prime}}=\frac{E_{2}}{\Delta_{*}}\left(v_{12}+v_{13} v_{32}\right) ; \\
C^{2^{\prime \prime 2} 2^{\prime \prime 2} 2^{\prime \prime}}=\frac{E_{2}}{\Delta_{*}}\left(1-v_{13} v_{31}\right) ; C^{2^{\prime \prime 2} 2^{\prime \prime} 3^{\prime \prime}}=\frac{E_{3}}{\Delta_{*}}\left(v_{23}+v_{21} v_{13}\right) ;
\end{aligned}
$$




$$
\begin{gathered}
C^{1^{\prime 2} 2^{\prime \prime} 1^{\prime \prime} 2^{\prime \prime}}=\frac{G_{12}}{2} ; \quad C^{2^{\prime \prime} 3^{\prime \prime} 2^{\prime \prime} 3^{\prime \prime}}=\frac{G_{23}}{2} ; \\
\Delta_{*}=\left(1-v_{23} v_{32}-v_{12} v_{21}-v_{12} v_{23} v_{31}-v_{13} v_{21} v_{32}-v_{13} v_{31}\right),
\end{gathered}
$$

where $E_{1}, E_{2}, E_{3}$ are elastic modules in corresponding directions of the reinforcement system, $G_{i j}$ are shear modules, $v_{i j}$ are Poisson's ratios.

It is assumed, that the coordinate system of finite element and the coordinate system of the reinforcement are transformed into one another by spatial rotation (Fig. 1). The connection between them is determined by relations:

Inverse connection can be written as

$$
\left\{\begin{array}{l}
x_{1}=x_{1^{\prime \prime}} \cos \alpha \cos \beta+x_{2^{\prime \prime}} \cos \alpha \sin \beta+x_{3^{\prime \prime}} \sin \alpha, \\
x_{2}=-x_{1^{\prime \prime}} \sin \beta+x_{2^{\prime \prime}} \cos \beta, \\
x_{3}=-x_{1^{\prime \prime}} \sin \alpha \cos \beta-x_{2^{\prime \prime}} \sin \alpha \sin \beta+x_{3^{\prime \prime}} \cos \alpha .
\end{array}\right.
$$

$$
\left\{\begin{array}{l}
x_{1^{\prime \prime}}=x_{1} \cos \alpha \cos \beta-x_{2} \sin \beta-x_{3} \sin \alpha \cos \beta, \\
x_{2^{\prime \prime}}=x_{1} \cos \alpha \sin \beta+x_{2} \cos \beta-x_{3} \sin \alpha \sin \beta, \\
x_{3^{\prime \prime}}=x_{1} \sin \alpha+x_{3} \cos \alpha
\end{array}\right.
$$

To convert the components of the tensor of the elastic constants from the coordinate system of the reinforcement $C^{i^{\prime \prime} j^{\prime \prime} k^{\prime \prime} l^{\prime \prime}}$ to finite element coordinates $C^{i j k l}$ can be written as:

$$
C^{i j k l}=C^{i^{\prime \prime} j^{\prime \prime} k^{\prime \prime} l^{\prime \prime}} a_{i^{\prime \prime}}^{i} a_{j^{\prime \prime}}^{j} a_{k^{\prime \prime}}^{k} a_{l^{\prime \prime}}^{l},
$$

where $a_{i^{\prime \prime}}^{i}=\frac{\partial x_{i}}{\partial x_{i^{\prime \prime}}}$ are the components of the tensor of the coordinate transformation. The matrix of the coordinate transformation has the form:

$$
[a]=\left[\begin{array}{ccc}
\cos \alpha \cos \beta & \cos \alpha \sin \beta & \sin \alpha \\
-\sin \beta & \cos \beta & 0 \\
-\sin \alpha \cos \beta & -\sin \alpha \sin \beta & \cos \alpha
\end{array}\right] .
$$

The approximation of the displacements inside of the finite element in basis coordinate system can be written as linear law:

$$
\begin{gathered}
u_{k^{\prime}}=\sum_{p=0}^{M} \sum_{q=0}^{N} \sum_{r=0}^{L} \omega_{k^{\prime}}^{(p q r)} \psi^{(p q r)}= \\
=\omega_{k^{\prime}}^{(000)}+\omega_{k^{\prime}}^{(100)} \psi^{(100)}+\omega_{k^{\prime}}^{(010)} \psi^{(010)}+\omega_{k^{\prime}}^{(001)} \psi^{(001)}+\omega_{k^{\prime}}^{(110)} \psi^{(110)}+\omega_{k^{\prime}}^{(101)} \psi^{(101)}+\omega_{k^{\prime}}^{(011)} \psi^{(011)}+\omega_{k^{\prime}}^{(111)} \psi^{(111)},
\end{gathered}
$$

where $\omega_{k^{\prime}}^{(p q r)}$ are the coefficients of the decomposition $\left(k^{\prime}=1,2,3\right) ; \psi^{(p q r)}=\frac{\left(x_{1}\right)^{p}\left(x_{2}\right)^{q}\left(x_{3}\right)^{r}}{p ! q ! r !}$ are the coordinate functions ( $p=0, \ldots, M ; q=0, \ldots, N ; r=0, \ldots, L$ are the degree of polynomial approximation).

By decomposing the components of the strain tensor in a Maclaurin series:

$$
\varepsilon_{i j}=\sum_{p=0}^{M} \sum_{q=0}^{N} \sum_{r=0}^{L} e_{i j}^{(p q r)} \psi^{(p q r)},
$$

where $e_{i j}^{(p q r)}$ are the coefficients of the decomposition.

In matrix form this decomposition can be written as:

$$
\left\{\varepsilon_{i j}\right\}=\left\{\psi_{i j}, y e_{i j}\right\} .
$$

The coefficients of the decomposition of the strain tensor defined in the center of the finite element can be written by the coefficients of the approximation of displacement $\omega_{k^{\prime}}^{(p q r)}$ as:

$$
\left\{e_{i j}\right\}=\left[F_{i j}^{k^{\prime}}\right]\left\{\omega_{k^{\prime}}\right\}
$$

For the variation of the elastic deformation energy using equations (11) and (2), we obtain:

$$
\delta W=\iiint_{V}\left\{\delta e_{i j}\right\}^{T}\left\{\psi_{i j}\right\}\left[C^{i j k l}\right]\left\{\psi_{k l}\right\}\left\{e_{k l}\right\} d V .
$$

The connection of the basic coordinate system with the coordinate system of the spatial isoparametric finite element with linear approximation of displacement is determined by relations:

$$
z_{k^{\prime}}=\sum_{L=0}^{8} N_{L}\left(x_{1}, x_{2}, x_{3}\right) z_{k^{\prime}}^{L},
$$

where $z_{k^{\prime}}^{L}$ is the $k^{\prime}$-coordinate of the $L$-node in basis coordinate system (Fig. 1), $k^{\prime}=1,2,3 ; L=1, \ldots, 8, N_{L}\left(x_{1}, x_{2}, x_{3}\right)$ are shape functions of the $L$-node, defined by the formula of the form [8]:

$$
N_{L}=\frac{1}{8}\left(1+x_{1} x_{1}^{L}\right)\left(1+x_{2} x_{2}^{L}\right)\left(1+x_{3} x_{3}^{L}\right),
$$


where $x_{i}^{L}$ is $i$-coordinate of the $L$-node in the coordinate system of the finite element (Fig. 1 ), $i=1,2,3 ; L=1, \ldots, 8$. Using the Cauchy relations can be written:

$$
\varepsilon_{i j}=\frac{1}{2}\left(\frac{\partial z_{m^{\prime}}}{\partial x_{j}} u_{m^{\prime}, i}+\frac{\partial z_{m^{\prime}}}{\partial x_{i}} u_{m^{\prime}, j}\right),
$$

and using the relations (8) and (13) can be written:

$$
\begin{gathered}
\varepsilon_{11}=\frac{\partial z_{k^{\prime}}}{\partial x_{1}}\left(\omega_{k^{\prime}}^{(100)}+\omega_{k^{\prime}}^{(110)} \psi^{(010)}+\omega_{k^{\prime}}^{(101)} \psi^{(001)}+\omega_{k^{\prime}}^{(111)} \psi^{(011)}\right), \varepsilon_{22}=\frac{\partial z_{k^{\prime}}}{\partial x_{2}}\left(\omega_{k^{\prime}}^{(010)}+\omega_{k^{\prime}}^{(110)} \psi^{(100)}+\omega_{k^{\prime}}^{(011)} \psi^{(001)}+\omega_{k^{\prime}}^{(111)} \psi^{(101)}\right), \\
\varepsilon_{33}=\frac{\partial z_{k^{\prime}}}{\partial x_{3}}\left(\omega_{k^{\prime}}^{(001)}+\omega_{k^{\prime}}^{(101)} \psi^{(100)}+\omega_{k^{\prime}}^{(011)} \psi^{(010)}+\omega_{k^{\prime}}^{(111)} \psi^{(110)}\right), \\
\varepsilon_{12}=\frac{1}{2}\left(\frac{\partial z_{k^{\prime}}}{\partial x_{2}}\left(\omega_{k^{\prime}}^{(100)}+\omega_{k^{\prime}}^{(110)} \psi^{(010)}+\omega_{k^{\prime}}^{(101)} \psi^{(001)}+\omega_{k^{\prime}}^{(111)} \psi^{(011)}\right)+\frac{\partial z_{k^{\prime}}}{\partial x_{1}}\left(\omega_{k^{\prime}}^{(010)}+\omega_{k^{\prime}}^{(110)} \psi^{(100)}+\omega_{k^{\prime}}^{(011)} \psi^{(001)}+\omega_{k^{\prime}}^{(111)} \psi^{(101)}\right)\right), \\
\varepsilon_{13}=\frac{1}{2}\left(\frac{\partial z_{k^{\prime}}}{\partial x_{3}}\left(\omega_{k^{\prime}}^{(100)}+\omega_{k^{\prime}}^{(110)} \psi^{(010)}+\omega_{k^{\prime}}^{(101)} \psi^{(001)}+\omega_{k^{\prime}}^{(111)} \psi^{(011)}\right)+\frac{\partial z_{k^{\prime}}}{\partial x_{1}}\left(\omega_{k^{\prime}}^{(001)}+\omega_{k^{\prime}}^{(101)} \psi^{(100)}+\omega_{k^{\prime}}^{(011)} \psi^{(010)}+\omega_{k^{\prime}}^{(111)} \psi^{(110)}\right)\right), \\
\varepsilon_{23}=\frac{1}{2}\left(\frac{\partial z_{k^{\prime}}}{\partial x_{3}}\left(\omega_{k^{\prime}}^{(010)}+\omega_{k^{\prime}}^{(110)} \psi^{(100)}+\omega_{k^{\prime}}^{(011)} \psi^{(001)}+\omega_{k^{\prime}}^{(111)} \psi^{(101)}\right)+\frac{\partial z_{k^{\prime}}}{\partial x_{2}}\left(\omega_{k^{\prime}}^{(001)}+\omega_{k^{\prime}}^{(101)} \psi^{(100)}+\right.\right. \\
\left.\left.+\omega_{k^{\prime}}^{(011)} \psi^{(010)}+\omega_{k^{\prime}}^{(111)} \psi^{(110)}\right)\right) .
\end{gathered}
$$

The coefficients of the decomposition $e_{i j}^{(p q r)}$ in series (9) is obtained by means of the formula

and has the form

$$
e_{i j}^{(p q r)}=\left.\frac{\partial^{p+q+r} \varepsilon_{i j}}{\left(\partial x_{1}\right)^{p}\left(\partial x_{2}\right)^{q}\left(\partial x_{3}\right)^{r}}\right|_{x_{1}=x_{2}=x_{3}=0}
$$

$$
\begin{aligned}
& e_{11}^{(p q r)}=\sum_{\mu \nu \eta}^{p q r} \omega_{k^{\prime}}^{(\mu+1 v \eta)} f_{(p+1-\mu q-v r-\eta)}^{k^{\prime}} ; \quad e_{22}^{(p q r)}=\sum_{\mu v \eta}^{p q r} \omega_{k^{\prime}}^{(\mu v+1 \eta)} f_{(p-\mu q+1-v r-\eta)}^{k^{\prime}} ; \\
& e_{33}^{(p q r)}=\sum_{\mu \nu \eta}^{p q r} \omega_{k^{\prime}}^{(\mu \nu \eta+1)} f_{(p-\mu q-v r+1-\eta)}^{k^{\prime}} ; \quad e_{12}^{(p q r)}=\frac{1}{2} \sum_{\mu \nu \eta}^{p q r}\left(\omega_{k^{\prime}}^{(\mu \nu+1 \eta)} f_{(p-\mu+1 q-v r-\eta)}^{k^{\prime}}+\omega_{k^{\prime}}^{(\mu+1 v \eta)} f_{(p-\mu q-v+1 r-\eta)}^{k^{\prime}}\right) \text {; } \\
& e_{13}^{(p q r)}=\frac{1}{2} \sum_{\mu \nu \eta}^{p q r}\left(\omega_{k^{\prime}}^{(\mu \nu \eta+1)} f_{(p-\mu+1 q-v r-\eta)}^{k^{\prime}}+\omega_{k^{\prime}}^{(\mu+1 v \eta)} f_{(p-\mu q-v r-\eta+1)}^{k^{\prime}}\right) ; \\
& e_{23}^{(p q r)}=\frac{1}{2} \sum_{\mu \nu \eta}^{p q r}\left(\omega_{k^{\prime}}^{(\mu v \eta+1)} f_{(p-\mu q-v+1 r-\eta)}^{k^{\prime}}+\omega_{k^{\prime}}^{(\mu v+1 \eta)} f_{(p-\mu q-v r-\eta+1)}^{k^{\prime}}\right),
\end{aligned}
$$

where

$$
f_{(\mu \nu \eta)}^{k^{\prime}}=\left.\frac{\partial^{\mu+v+\eta} z_{k^{\prime}}}{\left(\partial x_{1}\right)^{\mu}\left(\partial x_{2}\right)^{v}\left(\partial x_{3}\right)^{\eta}}\right|_{x_{1}=x_{2}=x_{3}=0} .
$$

It should be pointed out that not all of the coefficients $\omega_{k^{\prime}}^{(p q r)}$ in the relations (18) are present in the expansion of the displacement (8). Such coefficients should be excluded from the decomposition (9). Then the components of the strain tensor have the form:

$$
\begin{aligned}
& \varepsilon_{11}=e_{11}^{(000)}+e_{11}^{(010)} \psi^{(010)}+e_{11}^{(001)} \psi^{(001)}+e_{11}^{(011)} \psi^{(011)}, \\
& \varepsilon_{22}=e_{22}^{(000)}+e_{22}^{(100)} \psi^{(100)}+e_{22}^{(001)} \psi^{(001)}+e_{22}^{(101)} \psi^{(101)}, \\
& \varepsilon_{33}=e_{33}^{(000)}+e_{33}^{(100)} \psi^{(100)}+e_{33}^{(010)} \psi^{(010)}+e_{33}^{(110)} \psi^{(110)}, \\
\varepsilon_{12}= & e_{12}^{(000)}+e_{12}^{(001)} \psi^{(001)}, \varepsilon_{13}=e_{13}^{(000)}+e_{13}^{(010)} \psi^{(010)}, \quad \varepsilon_{23}=e_{23}^{(000)}+e_{23}^{(100)} \psi^{(100)} .
\end{aligned}
$$

We form the matrix $\left[F_{i j}^{k^{\prime}}\right]$, which is a part of relation (11), with regard to the relations (18), (20). Then, taking into account relations (10) and (11), elastic energy variation in a matrix form takes the form:

$$
\delta W=\iint_{V}\left\{\delta \omega_{k^{\prime}}\right\}^{T}\left[F_{i j}^{k^{\prime}}\right]^{T}\left\{\psi_{i j}\right\}^{T}\left[C^{i j k l}\right\}\left\{\psi_{k l}\right\}\left[F_{k l}^{m^{\prime}}\right]\left\{\omega_{m^{\prime}}\right\} d V .
$$

or

$$
\delta W=\left\{\delta \omega_{k^{\prime}}\right\}^{T}\left[F_{i j}^{k^{\prime}}\right]^{T}\left[H ^ { i j k l } [ F _ { k l } ^ { m ^ { \prime } } ] \left\{\left(\omega_{m^{\prime}}\right\},\right.\right.
$$

where $\left[H^{i j k l}\right]=\int_{-1}^{1} \int_{-1}^{1} \int_{-1}^{1}\left\{\psi_{i j}\right\}^{T}\left[C^{i j k l}\right]\left\{\psi_{k l}\right\} \sqrt{g} d x_{1} d x_{2} d x_{3}$ is a matrix of elastic constants characterizing the physical properties of anisotropic material, taking into account the metric of space. 
For isoparametric finite element the components of the displacement vector are determined by the values of the nodal displacement vector and shape functions (14) as

$$
u_{k^{\prime}}=\sum_{L=0}^{8} N_{L}\left(x_{1}, x_{2}, x_{3}\right) u_{k^{\prime}}^{L},
$$

where $u_{k^{\prime}}^{L}$ is displacement of the $L$-node along $k^{\prime}$-direction of the basic coordinate system.

By means of transformation matrix $[A]$ we turn from coefficients $\omega_{k^{\prime}}^{(p q r)}$ to nodal vales of displacements $u_{k^{\prime}}^{L}$ in relation (8). The matrix $[A]$ sets relations between approximating Lagrange functions (shape functions $N_{L}$ ) and power functions $\psi^{(p q r)}$. The approximation of the displacements (8) inside of the finite element in basis coordinate system can be written as:

$$
u_{k^{\prime}}=\left\{\psi_{i j}^{*}\right\}\left\{\omega_{k^{\prime}}^{*}\right\}^{T},
$$

where $\left\{\omega_{k^{\prime}}^{*}\right\}$ is the 8-dimentional vector of the decomposition coefficients $\omega_{k^{\prime}}^{(p q r)},(p, q, r=0,1)$, $\left\{\psi_{i j}^{*}\right\}=\left\{1, x_{1}, x_{2}, x_{1} x_{2}, x_{3}, x_{1} x_{3}, x_{2} x_{3}, x_{1} x_{2} x_{3}\right\}$ is the vector of the power functions.

On the other hand, according to (23) we have in matrix form:

$$
u_{k^{\prime}}=\left\{N_{L}\right\}\left\{u_{k^{\prime}}^{*}\right\}^{T},
$$

where $\left\{u_{k^{\prime}}^{*}\right\}=\left\{u_{k^{\prime}}^{(1)}, u_{k^{\prime}}^{(2)}, u_{k^{\prime}}^{(3)}, u_{k^{\prime}}^{(4)}, u_{k^{\prime}}^{(5)}, u_{k^{\prime}}^{(6)}, u_{k^{\prime}}^{(7)}, u_{k^{\prime}}^{(8)}\right\}$ is the vector of the node displacement, $\left\{N_{L}\right\}=\left(N_{1}, N_{2}, N_{3}, N_{4}, N_{5}, N_{6}, N_{7}, N_{8}\right)$ is the vector of the shape functions determined by formula (14).

The matrix $[A]$ is determined on the base of taken law of displacement approximation. The shape functions can be written as follows by means of power functions:

$$
\left\{N_{L}\right\}=\left\{\psi_{i j}^{*}\right\}[A *]^{T},
$$

where $\left[A^{*}\right]$ is submatrix of matrix $[A]$, which is related to functions (14).

Then, taking into account the equality of the right parts of relations (24) and (25), we obtain:

or in general case:

$$
\left\{\omega_{k^{\prime}}^{*}\right\}^{T}=[A *]^{T}\left\{u_{k^{\prime}}^{*}\right\}^{T},
$$

$$
\left\{\omega_{k^{\prime}}\right\}=[A]^{T}\left\{u_{k^{\prime}}^{L}\right\} .
$$

Thus, variation of the elastic deformation energy according to formula (28) can be written as:

$$
\delta W=\left\{\delta u_{k^{\prime}}^{L}\right\}^{T}[A]\left[F_{i j}^{k^{\prime}}\right]^{T}\left[H^{i j k l}\right]\left[F_{k l}^{m^{\prime}}\right][A]^{T}\left\{u_{m^{\prime}}^{L}\right\},
$$

or

$$
\delta W=\left\{\delta u_{k^{\prime}}^{L}\right\}^{T}\left[K^{k^{\prime} m^{\prime}}\right]\left\{u_{m^{\prime}}^{L}\right\} .
$$

The stiffness matrix of a finite element is obtained by means of the moment scheme for spatially reinforced composite has the form:

$$
\left[K^{k^{\prime} m^{\prime}}\right]=[A]\left[F_{i j}^{k^{\prime}}\right]^{T}\left[H^{i j k l}\right]\left[F_{k l}^{m^{\prime}}\right][A]^{T} .
$$

The dimension of the stiffness matrix is $24 \times 24$.

The above approach to the construction of finite element stiffness matrix is implemented in the software package «MIPEЛA+».

Let us research convergence of numerical calculation results based on the finite element stiffness matrix. Consider an axially symmetric problem for a hollow cylinder made of the fiber composite under the internal pressure and external cylindrical surface clamped. General analytical solution of the plane problem for a homogeneous orthotropic material is presented in [9]. For radial displacements we have:

$$
u_{r}=A r^{\sqrt{\frac{E_{\theta}}{E_{r}}}}+B / r^{\sqrt{\frac{E_{\theta}}{E_{r}}}}
$$

where $E_{r}, E_{\theta}$ are orthotropic elastic modules in the radial and tangential direction.

The constants $A$ and $B$ can be found taking into account conditions $\sigma_{r}\left(r_{1}\right)=-q, u_{r}\left(r_{2}\right)=0$, where $r_{1}$ and $r_{2}$ are inner and outer radius of the cylinder, $q$ is internal pressure. Then

$$
u_{r}=A\left(r^{\sqrt{\frac{E_{\theta}}{E_{r}}}}-r_{2}^{2 \sqrt{\frac{E_{\theta}}{E_{r}}}} / r^{\sqrt{\frac{E_{\theta}}{E_{r}}}}\right), A=\frac{-q r_{1}^{\sqrt{\frac{E_{\theta}}{E_{r}}}+1}\left(1-v_{r \theta} v_{\theta r}\right)}{r_{2}^{2 \sqrt{\frac{E_{\theta}}{E_{r}}}}\left(\sqrt{E_{r} E_{\theta}}-E_{\theta} v_{r \theta}\right)+r_{1}^{2} \sqrt{\frac{E_{\theta}}{E_{r}}}\left(\sqrt{E_{r} E_{\theta}}+E_{\theta} v_{r \theta}\right)},
$$

where $v_{r \theta}, v_{\theta r}$ are Poisson's ratios.

In the reinforcement coordinate system the composite material is represented as transtropic homogeneous material on the basis of the mixture rule:

$$
E_{1^{\prime \prime}}=E^{*}(1-f)+E^{\circ} f, E_{2^{\prime \prime}}=\frac{E^{*} E^{\circ}}{E^{*} f+E^{\circ}(1-f)}, E_{2^{\prime \prime}}=E_{3^{\prime \prime}},
$$




$$
\begin{gathered}
v_{1^{\prime \prime} 2^{\prime \prime}}=v^{\circ} f+v^{*}(1-f), v_{2^{\prime \prime} 1^{\prime \prime}}=\frac{E_{2^{\prime \prime}}}{E_{1^{\prime \prime}}} v_{1^{\prime \prime} 2^{\prime \prime}}, \\
G_{2^{\prime \prime} 3^{\prime \prime}}=G^{*} \frac{g+f+(1-f) G^{*} / G^{\circ}}{g(1-f)+(1+f g) G^{*} / G^{\circ}}, v_{2^{\prime \prime} 3^{\prime \prime}}=\frac{E_{2^{\prime \prime}}}{2 G_{2^{\prime \prime} 3^{\prime \prime}}}-1 \quad v_{2^{\prime \prime} 3^{\prime \prime}}=v_{3^{\prime \prime} 2^{\prime \prime}} .
\end{gathered}
$$

Here $E^{*}, G^{*}, v^{*}$ are elastic module, shear modulus and Poisson's ratio of the matrix material, $E^{\circ}, G^{\circ}, v^{\circ}$ are elastic module, shear modulus and Poisson's ratio of the fiber material, $f$ is the volume of the fiber in composite, $g=3-4 v^{*}$.

For axially symmetric problem may be three variants of the reinforcement:

-in tangentional direction, then $E_{r}=E_{2^{\prime \prime}}, E_{\theta}=E_{1^{\prime \prime}}, v_{r \theta}=v_{2^{\prime \prime} 1^{\prime \prime}}, v_{\theta r}=v_{1^{\prime 2} 2^{\prime \prime}}$;

- in axial direction, then $E_{r}=E_{2^{\prime \prime}}, E_{\theta}=E_{3^{\prime \prime}}, v_{r \theta}=v_{2^{\prime \prime} 3^{\prime \prime}}, v_{\theta r}=v_{3^{\prime \prime 2} 2^{\prime \prime}}$;

- in radial direction, then $E_{r}=E_{1^{\prime \prime}}, E_{\theta}=E_{2^{\prime \prime}}, v_{r \theta}=v_{1^{\prime \prime 2} 2^{\prime \prime}}, v_{\theta r}=v_{2^{\prime \prime} 1^{\prime \prime}}$.

\section{NUMERICAL RESULT}

As an example of the above method the calculation of displacement is performed with the following parameters of the matrix and the fiber. It is assumed, that the matrix material is a rubber, its $E^{*}=4,4 \mathrm{MPa} ; v^{*}=0,49$. The fiber is polyamide cord with the following parameters: $E^{\circ}=1277,5 \mathrm{MPa} ; v^{\circ}=0,3$, inner radius of the cylinder $r_{1}=0,1 \mathrm{~m}$, outer radius of the cylinder $r_{2}=0,15 \mathrm{~m}$, internal pressure $q=5 \mathrm{MPa}$. The result of calculations for different discretization meshes show stable convergence to analytical solution. The results of calculations for discretization mesh $10 \times 21 \times 3$ are shown in Table. Relative error of the numerical solution is calculated by means of the formula: $\varepsilon=\frac{u_{n}-u_{a}}{u_{a}} 100 \%$, where $u_{a}$ is analytical solution by means of the formula (32), $u_{n}$ is numerical solution by means of the moment schema of the finite element.

Table

\begin{tabular}{|c|c|c|c|c|c|c|}
\hline \multirow{3}{*}{$\begin{array}{l}\text { Volume of the } \\
\text { fiber in composite } \\
f\end{array}$} & \multicolumn{6}{|c|}{ Reinforcement } \\
\hline & \multicolumn{2}{|c|}{ in tangentional direction } & \multicolumn{2}{|c|}{ in radial direction } & \multicolumn{2}{|c|}{ in axial direction } \\
\hline & $\begin{array}{c}\text { Analytical } \\
\text { solution by means } \\
\text { of the formula } \\
(32), \mathrm{m}\end{array}$ & $\begin{array}{c}\text { Numerical } \\
\text { solution by means } \\
\text { of the moment } \\
\text { schema of the } \\
\text { finite element, } \mathrm{m} \\
\text { (relative error, \%) }\end{array}$ & $\begin{array}{c}\text { Analytical } \\
\text { solution by means } \\
\text { of the formula } \\
(32), \mathrm{m}\end{array}$ & $\begin{array}{c}\text { Numerical } \\
\text { solution by means } \\
\text { of the moment } \\
\text { schema of the } \\
\text { finite element, } \mathrm{m} \\
\text { (relative error, \%) }\end{array}$ & $\begin{array}{c}\text { Analytical } \\
\text { solution by means } \\
\text { of the formula } \\
(32), \mathrm{m}\end{array}$ & $\begin{array}{c}\text { Numerical } \\
\text { solution by means } \\
\text { of the moment } \\
\text { schema of the } \\
\text { finite element, } \mathrm{m} \\
\text { (relative error, \%) }\end{array}$ \\
\hline 0 & $4.093 \times 10^{-2}$ & $\begin{array}{c}3.759 \times 10^{-2} \\
(-8.16 \%)\end{array}$ & $4.093 \times 10^{-2}$ & $\begin{array}{c}3.759 \times 10^{-2} \\
(-8.16 \%)\end{array}$ & $4.093 \times 10^{-2}$ & $\begin{array}{c}3.759 \times 10^{-2} \\
(-8.16 \%)\end{array}$ \\
\hline 0.2 & $1.401 \times 10^{-2}$ & $\begin{array}{l}1.392 \times 10^{-2} \\
(-0.64 \%)\end{array}$ & $7.810 \times 10^{-4}$ & $\begin{array}{c}7.821 \times 10^{-4} \\
(0,26 \%)\end{array}$ & $3.629 \times 10^{-2}$ & $\begin{array}{c}3.631 \times 10^{-2} \\
(0,06 \%)\end{array}$ \\
\hline 0.4 & $8.539 \times 10^{-3}$ & $\begin{array}{c}8.444 \times 10^{-3} \\
(-1.12 \%)\end{array}$ & $3.940 \times 10^{-4}$ & $\begin{array}{c}3.948 \times 10^{-4} \\
(0.20 \%)\end{array}$ & $2.694 \times 10^{-2}$ & $\begin{array}{c}2.696 \times 10^{-2} \\
(0.07 \%)\end{array}$ \\
\hline 0.6 & $5.684 \times 10^{-3}$ & $\begin{array}{c}5.634 \times 10^{-3} \\
(-0.88 \%)\end{array}$ & $2.640 \times 10^{-4}$ & $\begin{array}{c}2.640 \times 10^{-4} \\
(0.00 \%)\end{array}$ & $1.720 \times 10^{-2}$ & $\begin{array}{c}1.721 \times 10^{-2} \\
(0.06 \%)\end{array}$ \\
\hline 0.8 & $3.493 \times 10^{-3}$ & $\begin{array}{l}3.477 \times 10^{-3} \\
(-0.46 \%)\end{array}$ & $1.980 \times 10^{-4}$ & $\begin{array}{c}1.983 \times 10^{-4} \\
(0.15 \%)\end{array}$ & $8.203 \times 10^{-3}$ & $\begin{array}{c}8.207 \times 10^{-3} \\
(0.05 \%)\end{array}$ \\
\hline 1 & $1.550 \times 10^{-4}$ & $\begin{array}{l}1.539 \times 10^{-4} \\
(-0.71 \%)\end{array}$ & $1.550 \times 10^{-4}$ & $\begin{array}{l}1.539 \times 10^{-4} \\
(-0.71 \%)\end{array}$ & $1.550 \times 10^{-4}$ & $\begin{array}{l}1.539 \times 10^{-4} \\
(-0.71 \%)\end{array}$ \\
\hline
\end{tabular}

Displacement of the inner point of the cylinder in radial direction

Except for limiting cases ( $f=0$ and $f=1$ ), the smallest values of displacement are obtained with radial reinforcement, the largest - with axial reinforcement. It can be explained as follows. In case of radial reinforcement the radial stress is applied to more stiff fibers, whereas in case of axial reinforcement the radial stress is applied to less stiff material of the matrix. Tangential reinforcement gives the intermediate result.

\section{CONCLUSIONS}

Thus, the technique of constructing the stiffness matrix of the composite material with unidirectional fibers oriented spatially on the basis of the moment scheme of a finite element. It is based on double approximation of displacement components and deformations. The results of solution of the test problem with the help of the proposed finite element stiffness matrix show good agreement with the exact solutions. Calculation error, as a rule, does not exceed $1 \%$ for the composite material. And only for a homogeneous material (weakly compressible rubber) the error is $8 \%$. This is due to the necessity of taking into account the weak compressibility. One approach is the use of moment scheme of finite element for weakly compressible materials ${ }^{[1] .}$ 


\section{REFERENCES}

1. Kirichevskiy V.V. The finite element method in mechanics of elastomer. - K.: Naukova dumka, 2002. (in Russian).

2. Rikards R.B. The finite elements method in plates and shells theory. - Riga: Zinatne, 1988. (in Russian).

3. Reddy J.N. Mechanics of laminated composite plates and shells. Theory and analysis. - Boca Raton: CRC Press LLC, 2004.

4. Zienkiewicz O.C., Taylor R.L. The Finite Element Method for Solid and Structural Mechanics. - Oxford: Elsevier, 2005.

5. Tenek L.T., Argyris J. Finite Element Analysis for Composite Structures. - Dordrecht: Kluwer academic publishers, 1998.

6. Gopalakrishnan S., Chakraborty A., Mahapatra D.R. Spectral Finite Element Method: Wave Propagation, Diagnostics and Control in Anisotropic and Inhomogenous Structures. - London: Springer, 2008.

7. Matthews F.L., Davies G.A.O., Hitchings D., Soutis C. Finite element modelling of composite materials and structures. Cambridge: Woodhead Publishing, 2000.

8. Barbero E.J. Finite Element Analysis of Composite Materials (Composite Materials: Design and Analysis). - Boca Raton: CRC Press, 2007.

9. Bazhenov V.A., Gulyar A.I., Kozak A.L., Rutkowskij V.A., Sacharov A.S. Numerical modeling of destruction of concrete structures by the finite element method. - K.: Naukova dumka, 1996. (in Russian).

10. Bazhenov V.A., Sacharov A.S., Gondlyah A.V. Melnikov S.L. Nonlinear problems of mechanics of multilayer shells. - K.: NDI budmehaniki, 1994. (in Russian). 